\title{
EFFECT OF MECHANICAL AND BIOPHYSICAL PROPERTIES OF FIBRIN GEL ON MICROVASCUAL DEVELOPMENT IN A THREE-DIMENSONAL ANGIOGENESIS ASSAY
}

\author{
G. N. Vadival ${ }^{1}$ and I. Sukmana ${ }^{1,2}{ }^{*}$ \\ ${ }^{1}$ IJN-UTM Cardiovascular Engineering Center and Medical Devices Technology \\ Research Group (Mediteg), Faculty of Biosciences and Medical Engineering, \\ Block V01, Universiti Teknologi Malaysia, UTM Johor Bahru, 81310 Malaysia. \\ *Email: irza.sukmana@gmail.com \\ Phone: +6075558502; Fax: +6075559515 \\ ${ }^{2}$ Mechanical Engineering Department, Faculty of Engineering, University of Lampung, \\ Jl. Prof. Dr. Soemantri Brojonegoro 1, Bandar Lampung, 35143 Indonesia.
}

\begin{abstract}
This work aims to determine the mechanical and biophysical properties of fibrin gel for the purpose of angiogenesis study and microvascular development in a threedimensional (3D) environment. In the human body system, fibrin gel is known to be an extracellular matrix (ECM) that actively promotes homeostasis during blood vessel formation, wound healing and other inflammation processes. In this study, we first test the effect of fibrin and thrombin composition on mechanical and biophysical properties of the gel. Further in vitro experiments using human umbilical vein endothelial cells (HUVECs) were also conducted to find the optimum fibrinogen/thrombin concentration for the angiogenesis assay using a sandwich fibrin gel system. A fibrinogen/thrombin concentration of 2.0/1.0 results in optimum gel properties for three dimensional angiogenesis assay, which does not appear in other fibrinogen/thrombin concentrations. This sandwiched polymer fiber system has a potential future application for angiogenesis assay in tissue engineering and cardiovascular research.
\end{abstract}

Keywords: Mechanical properties; angiogenesis; three-dimensional assay; HUVECs.

\section{INTRODUCTION}

Fibrin gel is a biomaterial which is widely used in many various biomedical areas, including as bone tissue, cardiac tissue, nervous tissue and vascular graft and other tissues (Rao et al., 2012). This biodegradable material functions as matrices for the delivery and proliferation of living cells. Along with the development of tissue engineering and regenerative medicine, fibrin glue has been studied to find new applications (Clark, 2003; Jockenhoevel et al., 2001; Nakatsu \& Hughes, 2008). Among many biopolymers, fibrin appears to be one of the most commonly used hydrogel as it has the advantage that it can be obtained from and received by the same person and can thus avoid the risks of foreign body reaction and virus infection (Sukmana, 2012a). Although fibrin gel has been extensively applied in vitro and clinical, only in recent years has its significant value been recognized as a cell's extracellular matrices for angiogenesis studies. The structural and biochemical properties of the fibrin polymer also make it a promising candidate as scaffolding in tissue engineering and regenerative medicines (Laurens, Koolwijk, \& De Maat, 2006; 
Sukmana, 2012b). The modification and fictionalization of fibrin matrices has been used to provide control release growth factor to repair damaged tissues (Janmey, Winer, \& Weisel, 2009). Fibrin gel can be produced in vitro by mixing fibrinogen and thrombin solutions. Studies have shown that different concentrations of fibrinogen and thrombin affect the polymerization time of fibrin gel, therefore affecting its mechanical and physical properties. Due to this variation in mechanical and physical properties, fibrin gel can be manipulated to be used in various applications in the medical field. Some fibrin gel formulations lack specific mechanical properties compared to the native extracellular matrix (ECM), therefore strategies have been investigated for the improvement of their properties for broader application in tissue engineering and angiogenesis research (Blombäck \& Bark, 2004; Sukmana, 2012a). In this study we tested four different fibrinogen/thrombin concentrations then compared their mechanical and biophysical properties to find the optimum concentration for angiogenesis study.

\section{MATERIALS AND METHODS}

Hank's balanced salt solution (HBSS, H1387), fibrinogen (F4753) and thrombin (T4546) were purchased from Sigma-Aldrich Inc. (St Louis, USA). The fibrin gel was prepared by mixing $1 \mathrm{~mL}$ of fibrinogen solution $\left(4.0 \mathrm{mg} \mathrm{mL}^{-1}\right.$ in $\left.\mathrm{HBSS}\right)$ with $1 \mathrm{~mL}$ of a thrombin solution $\left(2 \mathrm{U} \mathrm{mL} \mathrm{m}^{-1}\right.$ in HBSS) to obtain a final concentration of fibrin to thrombin of 2.0/1.0. The solution was then placed on the bench for the final polymerization process, about 30 to 60 minutes at room temperature. Human umbilical vein endothelial cells (HUVECs) were purchased from Cambrex (cc-2519, Walkersville, MD USA). HUVECs were cultured at $37^{\circ} \mathrm{C}$ and $5 \% \mathrm{CO}_{2}$ in M199 culture medium (M5017, Sigma-Aldrich) supplemented with $100 \quad \mathrm{U} / 100 \quad \mu \mathrm{g} \quad \mathrm{mL}-1$ penicillin/streptomycin (15140-122, Invitrogen Corporation, NY, Grand Island, USA), $10 \%$ fetal bovine serum (FBS, F1051, Sigma-Aldrich), 2 mM L-glutamine (25030149, Invitrogen Corporation), and $15 \mathrm{mg} \mathrm{mL}-1$ endothelial growth factor supplement (ECGS, 356006, BD Biosciences, San Jose, CA, USA). HUVECs between passages 2 and 6 were used in all experiments. Fibrin gels were prepared based on four different concentrations of fibrinogen $(\mathrm{mg} / \mathrm{mL}$ ) to thrombin (Units/mL), (i.e., 2.0/1.0, 2.0/4.0, 0.5/4.0, and 4.0/0.5). For example, the concentration of 2.0/1.0 was prepared with $1 \mathrm{~mL} /$ well of fibrinogen solution $\left(4.0 \mathrm{mg} \mathrm{mL}^{-1}\right)$ in HBSS mixed with $1 \mathrm{~mL}$ of a thrombin solution $\left(2 \mathrm{UmL}^{-1}\right)$ in HBSS. The polymerization process of fibrinogen into fibrin is 5 min at room temperature followed by $20 \mathrm{~min}$ inside the incubator at $37^{\circ} \mathrm{C}$ and $5 \% \mathrm{CO}_{2}$. To test the water uptake, fibrin gel was soaked in saline solution at room temperature for the relevant time. For example, $1000 \mathrm{uL}$ of saline solution was soaked in fibrin gel, and the volume of PBS which does not diffuse to the gel was measured after 30 minutes. The same procedure was repeated for intervals of one hour, two hours and four hours and the values recorded. Turbidity is recorded for different time intervals using a UV-VIS Spectrophotometer (Shimadzu UV 1201) with wavelength 550nm. For SEM (Scanning Electron Microscopy) imaging, fibrin gel was first fixed with 4\% formaldehyde and rinsed three times with PBS. The fibrin gel was then freeze dried for $12 \mathrm{hrs}$ before being coated and observed under FESEM (Zeiss Supra 35Vp Gemini).

For the angiogenesis assay and imaging, HUVECs were first cultured for two days to cover the polymer monofilament fibers (i.e., poly-(ethylene terephthalate) PET). After the covering process, fiber-covered cells were then transferred to the new well plate and sandwiched between two fibrin gels. The step-by-step process of angiogenesis assay is presented in Figure 1. To observe endothelial cell morphology, 
samples were inspected daily under phase contrast microscopy. To prepare the fluorescence image, cell-seeded fibers were gently washed with PBS (3 times) and fixed in a formaldehyde solution $(3.75 \%, \mathrm{wt} / \mathrm{v})$ in PBS for $20 \mathrm{~min}$ then 3 times in PBS wash. Cells adhered to fibers were permeabilized with a Triton X-100 solution for $5 \mathrm{~min}$, and after rinsing, samples were incubated for $1 \mathrm{~h}$ in a PBS solution containing $20 \%(\mathrm{wt} / \mathrm{v})$ BSA. Samples were then incubated in a solution containing a mixture of TRITCphalloidin (1:300 dilution) and Hoechst 33258 (1:10,000 dilution) made in a blocking buffer solution containing $20 \%$ BSA for $1 \mathrm{~h}$ at room temperature in the dark and rinsed three times with PBS. Images of microvessels were taken using epi-fluorescence as well as biological Laser Scanning Confocal Microscope - LSCM (Olympus Fluoview FV300; Olympus Optical, Tokyo, Japan) equipped with an Olympus IX70 camera and recorded as high-resolution and as layer-by-layer files. A complete image reconstruction was made to illustrate microvessels in a 3D fashion. Images were edited with Image-Pro Plus Software to identify microvessels and lumen formation along the fiber axis.

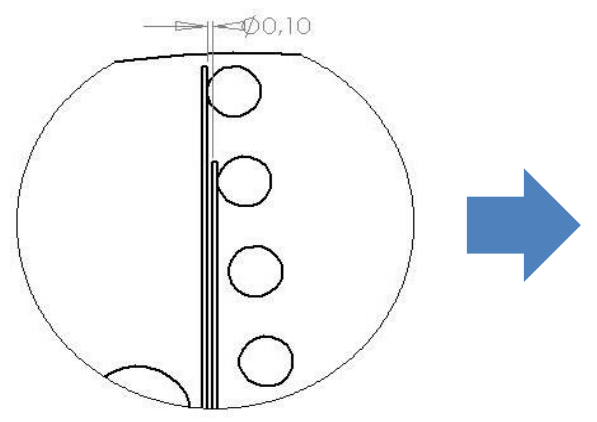

Fiber-frame

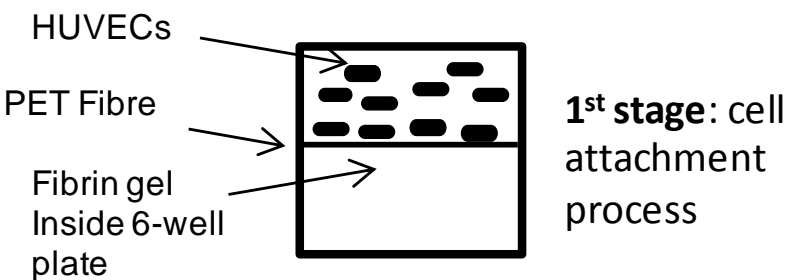

plate
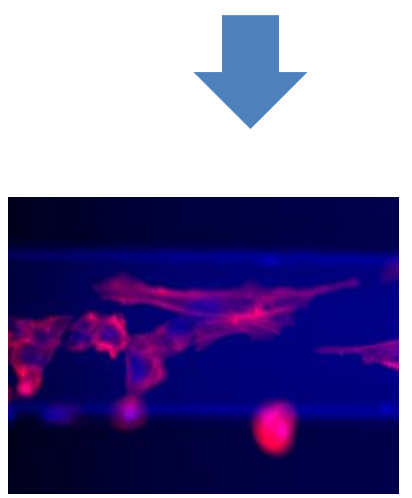

HUVECS

covered

PET fibres

HUVEC covered PET fibre

Figure 1. Schematic of a three-dimensional angiogenesis assay.

\section{RESULTS AND DISCUSSION}

Fibrin gel was easily formed by mixing solutions of fibrinogen and thrombin. Once completely clotted, the gel solution loses its fluidity and transforms into hydrogel. Clotting time highly affects the microstructure and handling properties of fibrin gel which is mostly controlled through the concentration of fibrinogen and thrombin (Blombäck \& Bark, 2004; Sukmana, 2011). The higher the thrombin composition, the faster the fibrin glue polymerizes. The concentration of 0.5/4.0 had the lowest clotting time of 45 seconds and the longest clotting time was for the concentration of 4.0/0.5, 540 seconds. Figure 2 shows the effect of fibrinogen/thrombin concentration on the mechanical and physical properties of fibrin gel. It shows that with an increase of 
thrombin concentration, clotting time decreases. Clotting time seems to be independent of the fibrinogen concentration (see Figure 2a). With an increase in thrombin concentration, more fibrinopeptides per unit time will be produced by the cleavage of fibrinogen, leading to a shorter clotting time. Other studies concluded that the addition of thrombin concentration increases hydrogen ion concentration, thus promoting faster shifting of ionic bonding during the conversion of fibrinogen to fibrin (Blombäck \& Bark, 2004).
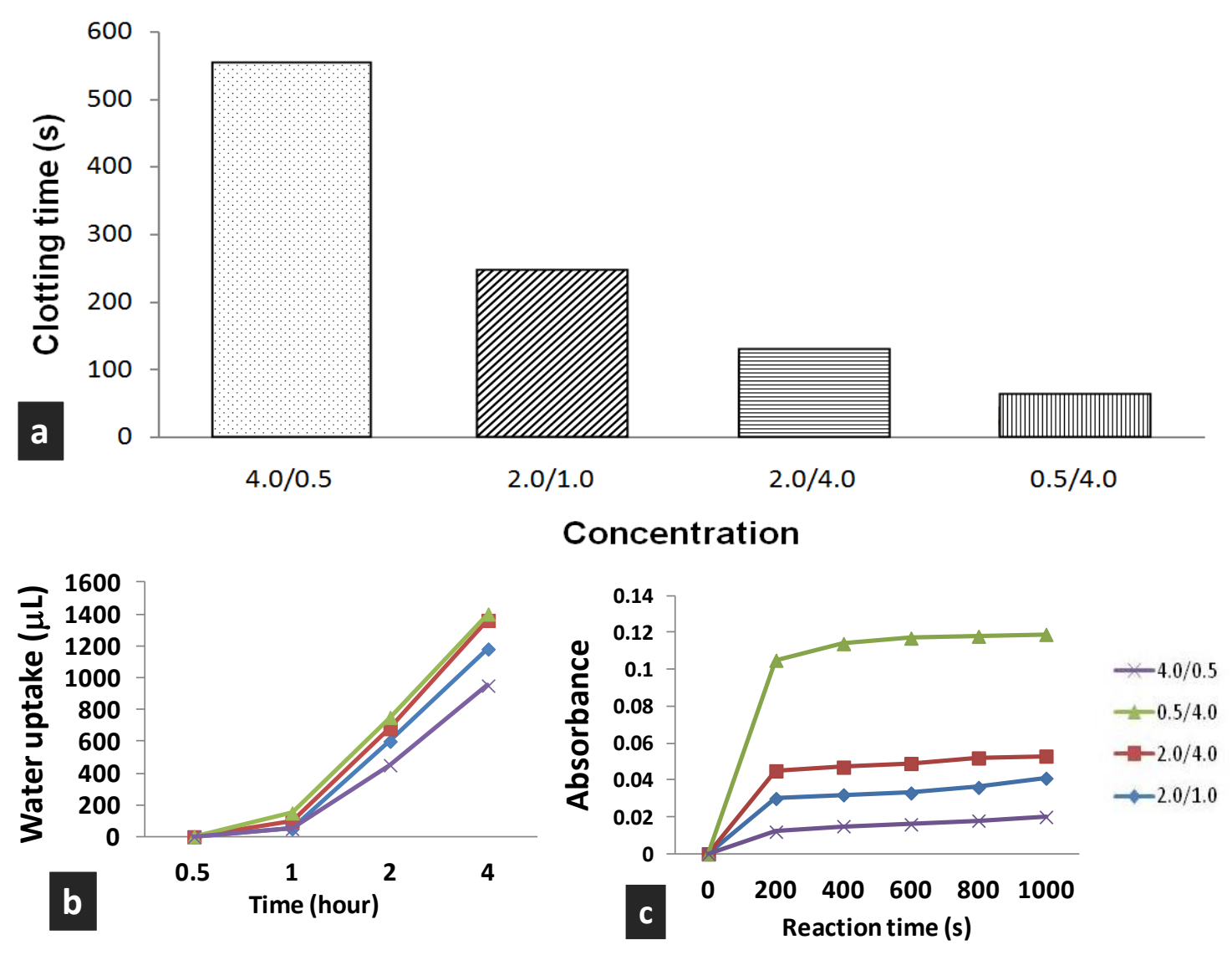

Figure 2. The effect of fibrinogen/thrombin concentration on the properties of fibrin gel: (a) Clotting time, (b) Water uptake (c) Absorbance.

Higher thrombin concentration results in better water uptake properties in the fibrin gel, shown in Figure 2b. A fibrinogen/thrombin concentration of 0.5/4.0 had the highest water uptake and 4.0/0.5 had the lowest. Higher thrombin concentration produces better cross-linking and a stronger ability to resist shrinkage, therefore more water can be absorbed compared to a lower thrombin concentration (Sukmana, 2011). Turbidity can be defined as the decrease in the transparency of a solution due to the presence of suspended and some dissolved substances, which causes incidental light to be scattered, reflected, and attenuated rather than transmitted in straight lines (Weisel, 2005). The concentration of $0.5 / 4.0$ has the highest turbidity, while $4.0 / 0.5$ has the lowest. With an increase in thrombin concentration, more fibrinopeptides per unit time will be produced by the cleavage of fibrinogen which prevents the light from moving in a straight line (Figure 2c). Turbidity is one of the most important parameters in designing scaffolds for in vitro tests in tissue engineering research. Higher turbidity will decrease the intensity of the fluorescence markers (Sukmana, 2012b). 
Changing fibrin/thrombin concentration also changed the microstructure of the fibrin micro-fibers property (Figure 3), as also presented by other studies (Litvinov, Gorkun, Owen, Shuman, \& Weisel, 2005). A concentration of 0.5/4.0 produced fibrin gel with a more dense structure and thick fibers. As the fibrinogen concentration increased $(2 \mathrm{mg} / \mathrm{mL})$, a slightly loose and porous structure was obtained. At $4 \mathrm{mg} / \mathrm{ml}$, the fibrinopeptides fibers become thinner and correspondingly looser. Along with the increase of thrombin concentration, the fibers become thinner but the change is not significant because little difference can be seen in Figure $3 b$, with a thrombin concentration of $1 \mathrm{U} / \mathrm{mL}$, and Figure $3 \mathrm{~d}$, with thrombin concentration of $4 \mathrm{U} / \mathrm{mL}$.
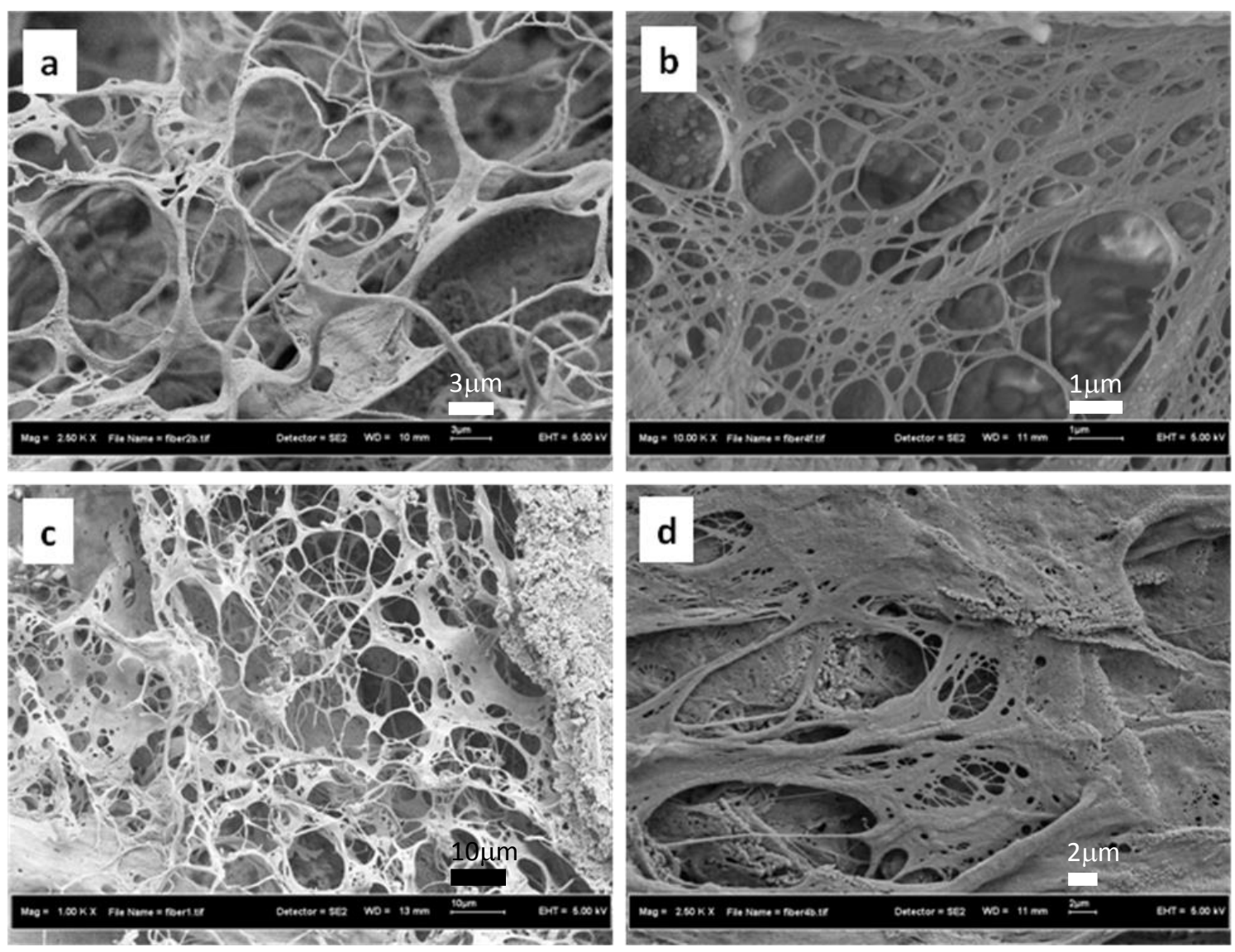

Figure 3. SEM images of the fibrin gel microstructures in four different fibrinogen/thrombin concentrations: (a) 2.0/4.0, (b) 4.0/0.5, (c) 2.0/1.0, and (d) 0.5/4.0.

Further test using HUVEC embedded in the gel shows that the concentration of 2.0/1.0 was the optimum composition for angiogenesis assays (see Figure 4). After a day of culture, some cells were able to build cell-to-cell connections along the fiber axis and even able to bridge between fibers, as shown on the phase contrast microscopy images (Figure 4a). After a further four days of culture, the cell-to-cell connection appears homogenously between two fibers (Figure 4b), and shows a tube-like structure of HUVEC and anastomosis development. The immunofluorescence images in Figure $4 \mathrm{a}$ and $4 \mathrm{~b}$ also confirmed the formation of cell-to-cell connections and tube-like structure development between two fibers. The maturation process of the patterned microvessel is also revealed when comparing the microvessel image of two days (Figure 4a) and four days of culture (Figure 4b). This 3D system, in which HUVEC-covered fibers embedded in the three-dimensional system containing HUVECs, allows a precise 
direction of microvessel and angiogenesis formation. A laser scanning confocal microscopy image of the sample after four days of experiment (Figure 4e) also confirmed the existence of tube-like structures between the PET fibers.
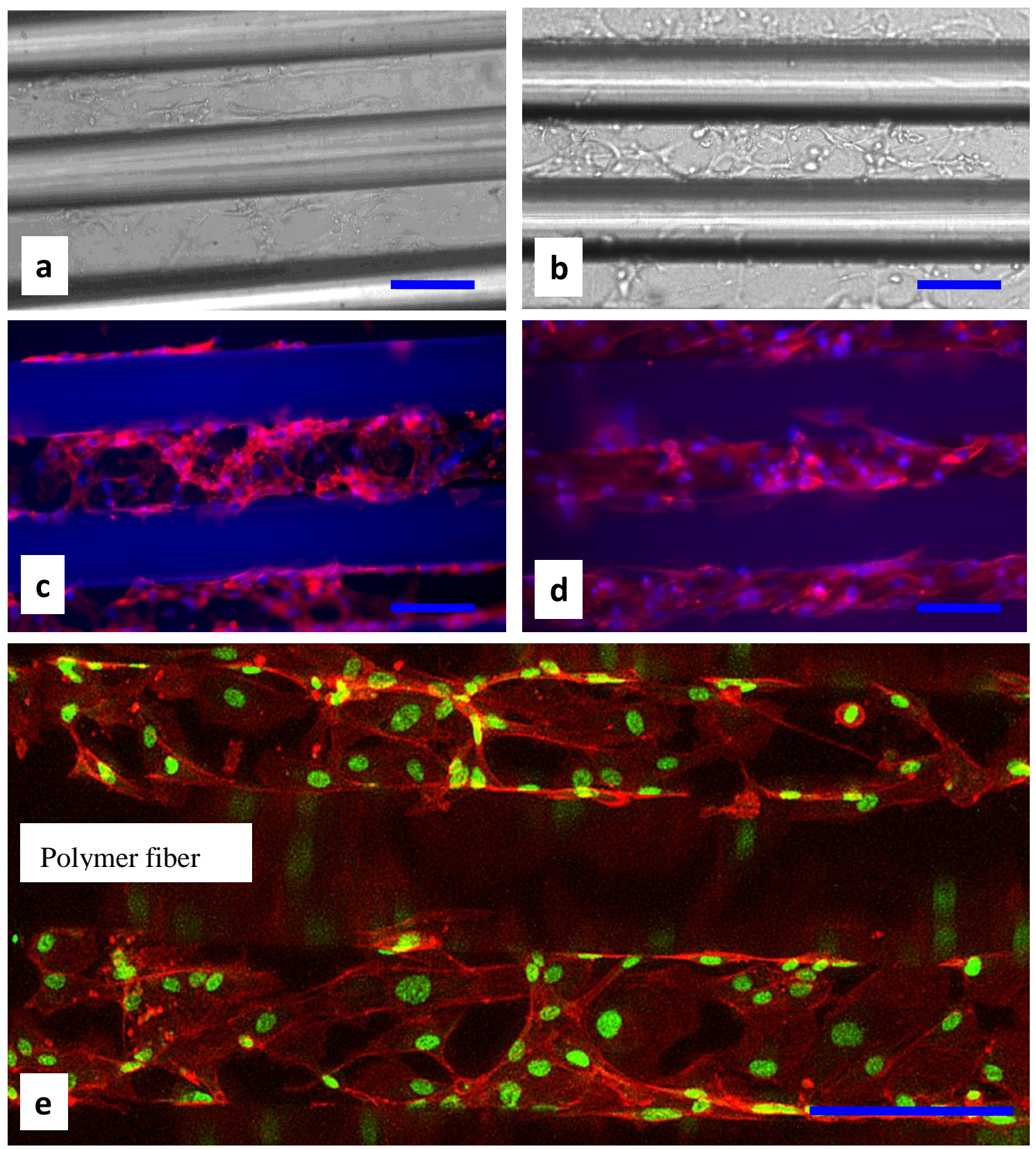

Figure 4. Angiogenesis test of the fibrin gel with concentration of 2.0/1.0. Images of HUVECs after 4 hours and 2 days of culture using: (a-b) phase contrast, and (c-d) epifluorescence, and (e) laser scanning confocal microscopy image. Bars are $100 \mu \mathrm{m}$.

\section{CONCLUSIONS}

Fibrinogen/thrombin concentration has a significant effect on fibrin gel properties. Thrombin concentration has more effect on the clotting time and turbidity of the gel, than fibrinogen. Microstructure observation demonstrated that fibrin hydrogels have a fibrous structure which is less sensitive to thrombin concentration. A 
fibrinogen/thrombin concentration of 2.0/1.0 resulted in optimum gel properties and angiogenesis development. After four days of culture, the cell-to-cell connection and tube-like structure appeared homogenously between two. This in vitro angiogenesis assay allows for broader applications in cardiovascular and tissue engineering research.

\section{ACKNOWLEDGEMENTS}

The author (IS) is supported by UTM RU Tier-1 (vote\# 03H12) and MOHE-FRGS (vote\# 4F128) research grants. The authors are grateful to Professor Patrick Vermette, University of Sherbrooke, QC, Canada for his scientific comments.

\section{REFERENCES}

Blombäck, B., \& Bark, N. (2004). Fibrinopeptides and fibrin gel structure. Biophysical Chemistry, 112(2), 147-151.

Clark, R. A. (2003). Fibrin glue for wound repair: Facts and fancy. Thrombosis and Haemostasis, 90(6), 1003-1006.

Janmey, P. A., Winer, J. P., \& Weisel, J. W. (2009). Fibrin gels and their clinical and bioengineering applications. Journal of the Royal Society Interface, 6(30), 1-10.

Jockenhoevel, S., Zund, G., Hoerstrup, S. P., Chalabi, K., Sachweh, J. S., Demircan, L.et al.Turina, M. (2001). Fibrin gel-advantages of a new scaffold in cardiovascular tissue engineering. European Journal of Cardio-thoracic Surgery, 19(4), 424-430.

Laurens, N., Koolwijk, P., \& De Maat, M. (2006). Fibrin structure and wound healing. Journal of Thrombosis and Haemostasis, 4(5), 932-939.

Litvinov, R. I., Gorkun, O. V., Owen, S. F., Shuman, H., \& Weisel, J. W. (2005). Polymerization of fibrin: Specificity, strength, and stability of knob-hole interactions studied at the single-molecule level. Blood, 106(9), 2944-2951.

Nakatsu, M. N., \& Hughes, C. C. (2008). An optimized three-dimensional in vitro model for the analysis of angiogenesis. Methods Enzymol, 443, 65-82.

Rao, R. R., Peterson, A. W., Ceccarelli, J., Putnam, A. J., \& Stegemann, J. P. (2012). Matrix composition regulates three-dimensional network formation by endothelial cells and mesenchymal stem cells in collagen/fibrin materials. Angiogenesis, 15(2), 253-264.

Sukmana, I. (2011). Imaging tools for the analysis of microvessel and angiogenesis development in a tridimensional cell culture system. 2nd International Conference on Instrumentation Control and Automation, pp. 181 - 183.

Sukmana, I. (2012a). Bioactive polymer scaffold for fabrication of vascularized engineering tissue. Journal of Artificial Organs, 15(3), 215-224.

Sukmana, I. (2012b). Microvascular guidance: A challenge to support the development of vascularised tissue engineering construct. The Scientific World Journal, 2012.

Weisel, J. W. (2005). Fibrinogen and fibrin. Advances in Protein Chemistry, 70, 247299. 